\title{
Production of a polarizable target using mechanical doping method and trial of dynamic nuclear polarization
}

\section{Daisuke Miura*, Takahiro Iwata, Yoshiyuki Miyachi, Genki Nukazuka and Daisuke Kaneko \\ Departument of Physics, Yamagata University, Kozirakawa-machi 1-4-12, Yamagata, 990-8560, Japan \\ E-mail: daisuke@quark.kj.yamagata-u.ac.jp, tiwata@sci.kj.yamagata-u.ac.jp, miyachilsci.kj.yamagata-u.ac.jp}

\begin{abstract}
Dynamic Nuclear Polarization (DNP) which is often employed for polarized solid targets, needs free electron doping to transfer high electron polarization to target nuclei. A newly proposed "mechanical-doping" method was applied in preparation of a polarized target material. A solid material having been crushed into small particles with a diameter of about $20 \mathrm{~nm}$, is mixed with a free radical. This allows target nuclei to have more chance to interact with the free electrons or polarized nuclei surrounding the small particles. A first DNP test at a magnetic field of $2.5 \mathrm{~T}$ and a temperature of $0.9 \mathrm{~K}$ was performed with a sample of $\mathrm{LaF}_{3}$ processed in the mechanical-doping method to introduce a free radical TEMPO dispersed in ethanol. ${ }^{19} \mathrm{~F}$ polarizations of $3.3 \%$ and $2.7 \%$ were achieved. The obtained polarization enhancement for ${ }^{19} \mathrm{~F}$ demonstrated effectiveness of the new method.
\end{abstract}

XVII International Workshop on Polarized Sources, Targets \& Polarimetry 16-20 October 2017

Kaist, South Korea 


\section{Introduction}

Polarized solid targets relying on DNP have been employed in numerous nuclear and particle physics experiments so far. The basic idea of the DNP allowing a high polarization of nuclear spins is using a microwave field in a high magnetic field to transfer the high polarization of electron spins to the nuclear spins. An essential point with the DNP is free electron doping into a target material. Nowadays, two doping methods are typically adopted: chemical-doping and radiation-doping. In the chemical-doping, paramagnetic dopants (polarizing agents) such as free radical molecules are dissolved into a target material such as butanol, and the solution is solidified in liquid nitrogen. This method is valid for only liquid material which the dopant is soluble in.

Therefore, basically, it is not applicable to insoluble solid materials. In contrast, the radiation-doping is applicable to any solid materials and solidified gas. Irradiated by an electron beam at low temperatures materials are doped with free electrons originating from lattice defects. the method requires special environment with a suitable electron linac, an apparatus with careful tuning of the irradiation condition [2]. Hence it is not so simple.

Taking account of the disadvantages above, we have proposed a new complementary method, mechanical-doping, which is applicable to any solid materials. A target material is crushed into fine particles and mixed with free radicals. If the particle size is fine enough, one can expect nuclear polarization enhancement with help of DNP.

\section{Mechanical doping}

The mechanical-doping is based on a simple idea that DNP should work when target nuclei are located close to free electrons. It consists of three stages, (1) milling and separation followed by (2)mixing stage and (3) mixing a free radical. In the first stage, a solid material is ground into very fine particles with a diameter of, let's say, $10 \mathrm{~nm}$. The diameter is appropriately tuned taking account of effective volume in which a single radical molecule effectively polarizes the nuclear spins by DNP. The volume is evaluated from the suitable electron concentration for DNP which is about $10^{19} \mathrm{~cm}^{-3}$ as reported in literatures, for example see [3]. In the second stage, the particles are mixed with a free radical. Finally, the free radical molecules are expected to be embedded uniformly into space among the particles or on the surface of the particles as depicted in Figure.1. In order to make sure uniform distribution, the radicals, being dissolved in solution, is mixed with the particles: the space is filled with the radical-dissolved-solution. When we choose a hydrogen compound such as ethanol as the solution, the hydrogens located close to the radical molecules are expected to be polarized at first, and their polarization is diffused over the material including the target nuclei inside the particles. This method is expected to be applicable to any insoluble solid materials with a combination of various dopants.

In this attempt, consisting of ${ }^{139} \mathrm{La}$ with the spin of $7 / 2$ and ${ }^{19} \mathrm{~F}$ with the spin of $1 / 2$ is employed as a target material. ${ }^{139} \mathrm{La}$ is one of the candiates of a target nucleus for the T-Violation experiment at J-PARC [4]. Comparing ${ }^{19} \mathrm{~F}$ and ${ }^{139} \mathrm{La}$, the ${ }^{19} \mathrm{~F}$ has simple spin of the $1 / 2$ and much larger magnetic moment, its polarization is easily measured and a higher polarization in DNP is expected. In this first attempt in mechanical doping method, the ${ }^{19} \mathrm{~F}$ was chosen to be polarized in DNP. $\mathrm{LaF}_{3}$ with a melting point of $1490{ }^{\circ} \mathrm{C}$ is solid at room temperature and is insoluble to usual 


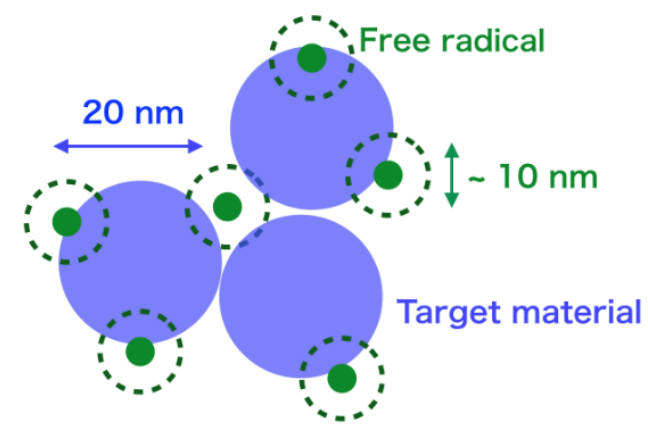

Figure.1: Schematic view of the target material prepared in the mechanical doping. The green dot circles show the area which radical polarize.

solutions such as alcohols, conventional chemical-doping cannot be applied. We produced the $\mathrm{LaF}_{3}$ sample doped with a free radical TEMPO (2,2,6,6-tetramethylpiperidine 1-oxyl) in order to study the effectiveness of DNP.

\subsection{Milling}

A ball-milling machine with a container and balls made of zirconium was employed to change a material into the small particles. $5 \mathrm{~g}$ of the $\mathrm{LaF}_{3}$ put in the container with the balls was milled. There are two milling methods, dry-milling, and wet-milling. In the former, the machine is run only with a material and balls, while in the latter some liquid is added to the container. The wet-milling is considered to give smaller particles than the other method. Comparing the two methods by actual test of milling, we chose the wet-milling. Two types of balls were used : balls with a diameter of $5 \mathrm{~mm}$ and $1 \mathrm{~mm}$. Table. 1 summarizes the final parameters of the milling. After the milling, the balls and $\mathrm{LaF}_{3}$ are taken out together and put into a centrifuge vessel with additional ethanol for the next step.

Table.1: Parameters inmilling

\begin{tabular}{|l|l|}
\hline Weigt of LaF3 [g] & 5 \\
\hline Method & Wet-millng (the solvent is ethanol about $5 \mathrm{ml})$ \\
\hline Balls & Blend $(2800$ balls of the diameter $1 \mathrm{~mm}$ and 160 balls of $5 \mathrm{~mm})$ \\
\hline Milling time [hours] & 9 \\
\hline
\end{tabular}




\subsection{Separation}

Although the milling step produced small particles with diameter down to $20 \mathrm{~nm}$, the sample is still contaminated with larger particles. They are separated by centrifuge. To obtain the $\mathrm{LaF}_{3}$ with a diameter less than $100 \mathrm{~nm}$, the centrifugation was performed at $2000 \mathrm{G}$ for 40 minutes. The particle size was evaluated with DLS (Dynamic Light Scattering) method5 ; the sample is irradiated with laser light, and fluctuation of the scattered laser intensity is measured. Since Stokes-Einstein law describes a relation between Brownian motion and the particle size, the fluctuation of the intensity gives a distribution of the particle size. The obtained distribution shows that the particle sizes after the separation are less than $100 \mathrm{~nm}$ in diameter, and the averaged diameter is $38 \mathrm{~nm}$ as shown in Figure. 2

\subsection{Mixing a free raical.}

The ethanol which is dominant in the skimmed sample was removed by heating. Since recombination of the small particles can occur if ethanol is evaporated completely, the heating procedure was terminated before the sample becomes completely dry. In this procedure $2 \mathrm{~g}$ of the sample including a small amount of ethanol was obtained. The sample was mixed with $60 \mathrm{mg}$ of liquid TEMPO by a stirrer. This gave an averaged spin density over the whole sample of $5.6 \times$ $10^{20} \mathrm{~cm}^{-3}$. It is notable that a much higher density is expected locally in the space among the particles filled with ethanol. The sample was put in a vessel made of glass and frozen with liquid nitrogen to be installed to the cryostat.

\section{Attempt of DNP}

A DNP test was carried out with the sample at a magnetic field of $2.5 \mathrm{~T}$ and temperature of $0.9 \mathrm{~K}$ with a refrigerator operated in ${ }^{4} \mathrm{He}$ mode. The polarization was obtained by measuring a signal area of CW-NMR, calibrated by measurements of a thermal equilibrium (TE) signal. In this attempt, we measured polarization of only ${ }^{1} \mathrm{H}$ in ethanol and ${ }^{19} \mathrm{~F}$ in $\mathrm{LaF}_{3}$. The NMR frequency at $2.5 \mathrm{~T}$ of ${ }^{1} \mathrm{H}$ and ${ }^{19} \mathrm{~F}$ are $106 \mathrm{MHz}$ and $100 \mathrm{MHz}$, respectively. Firstly, ${ }^{1} \mathrm{H}$ polarization was enhanced as shown in Figure. 3. The gray curves represent NMR signals in TE while the red and blue curves show the highest polarized ones. Positive polarization of 5.8\% and negative polarization of $-4.0 \%$ were achieved.

Then, ${ }^{19} \mathrm{~F}$ polarization was the enhanced as shown in Figure. 4. As the same the NMR signals of $1 \mathrm{H}$ in the Figure. 3, the gray curves mean TE signal, end others mean enhanced signals by DNP. This result can lead that A is effective for making DNP samples in solid. The ${ }^{19} \mathrm{~F}$ positive polarization of $3.3 \%$ and he negative polarization of $-2.7 \%$ were obtained. The Table. 2 summarizes the DNP test. It is notable that the obtained ${ }^{19} \mathrm{~F}$ polarizations are as high as two-thirds of the ${ }^{1} \mathrm{H}$ polarizations. Figure. 5 shows ${ }^{19} \mathrm{~F}$ polarization as a function of microwave frequency. The maximum polarization was obtained at the frequency of $69.95 \mathrm{GHz}$, and the minimum polarization was $70.30 \mathrm{GHz}$.

Relaxation time $T_{l}$ was measured for both nuclides at temperatures of $1.0 \mathrm{~K}$ and $1.6 \mathrm{~K}$ as shown in Table. 3. The $T_{l}$ for ${ }^{1} \mathrm{H}$ is rather short due to high electron density in the ethanol. On 


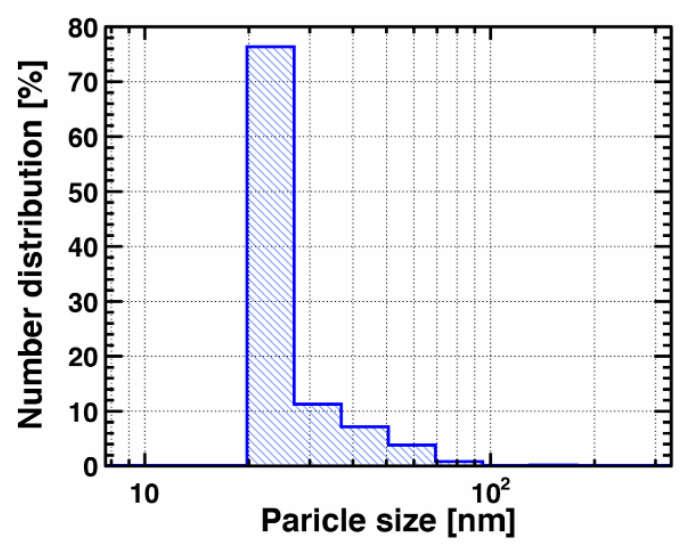

Figure.2: The number distibution after milling and separation steps. The average of particle diameter $38 \mathrm{~nm}$.
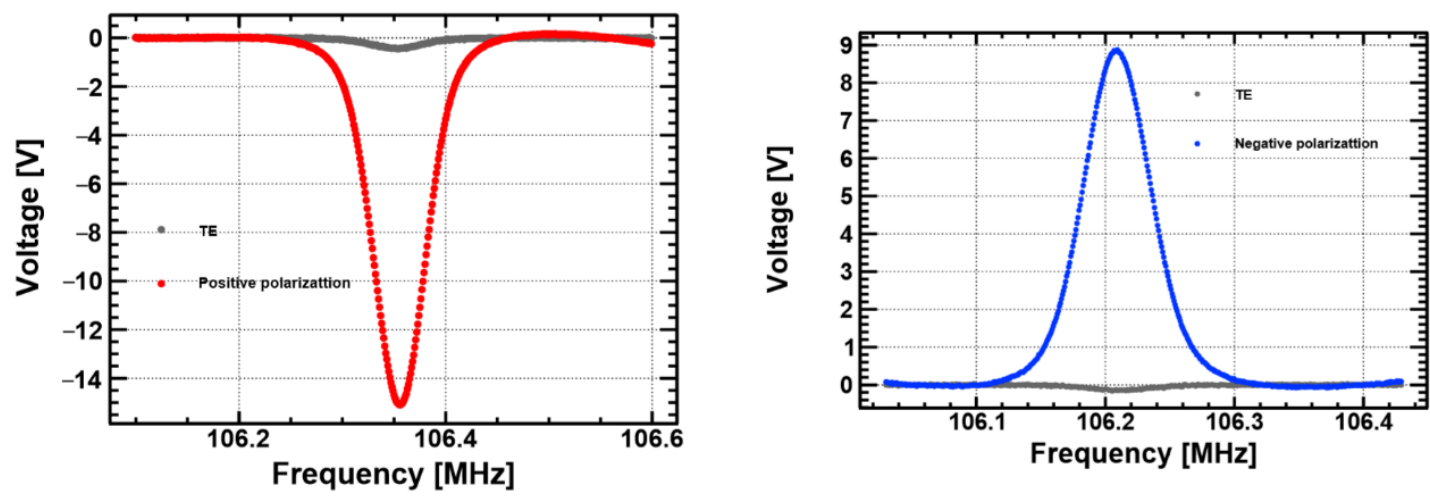

Figure.3: Left: The NMR signals of ${ }^{1} \mathrm{H}$ in TE and DNP for positive polarization.

Right ; The NMR signals of ${ }^{1} \mathrm{H}$ in TE anbd DNP for negative polarization.
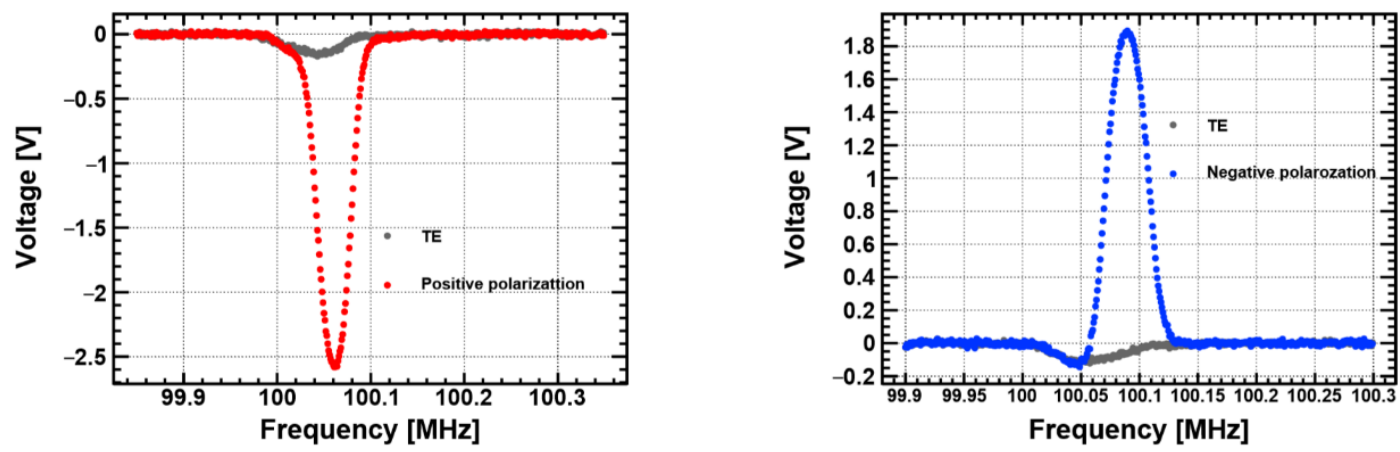

Figure.4: Left: The NMR signals of ${ }^{19} \mathrm{~F}$ in TE and DNP for positive polarization. Right: The NMR signals of ${ }^{19} \mathrm{~F}$ in TE and DNP for negative polarization. 


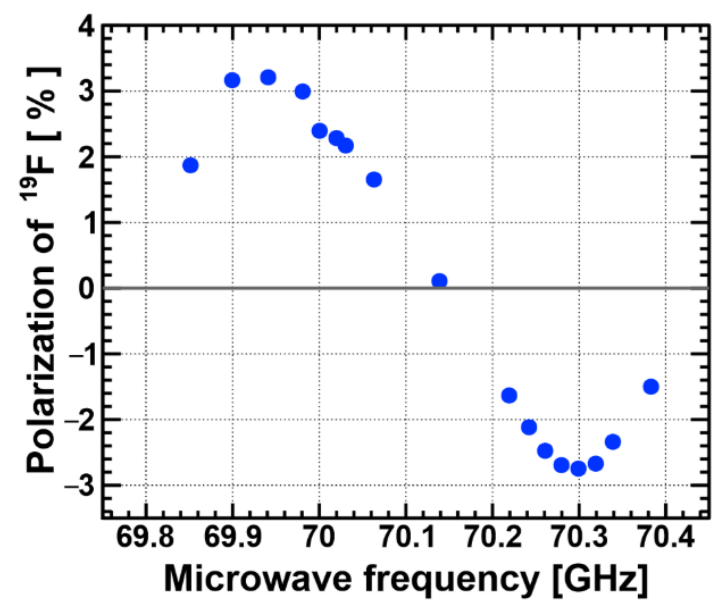

Figure.5: The polarization of ${ }^{19} \mathrm{~F}$ as a function of microwave frequency. The maximum polarization were at $69.98 \mathrm{GHz}$ and the minimum polarization at $70.22 \mathrm{GHz}$ obtained.

the contrary, ${ }^{19} \mathrm{~F}$ gave one order of magnitude longer values than ${ }^{1} \mathrm{H}$, because ${ }^{19} \mathrm{~F}$ nuclei are isolated farther from the free electrons. In future studies of the mechanical-doping method, the electron spin density is to be optimized in order to obtain higher polarizations.

Table.2: The sumarize of polarization of ${ }^{1} \mathrm{H}$ and ${ }^{19} \mathrm{~F}$.

\begin{tabular}{c|c|c|c}
\hline Nuclei & Direction & $\begin{array}{c}\text { Polarization } \\
{[\%]}\end{array}$ & $\begin{array}{c}\text { Frequency } \\
{[\mathrm{GHz}]}\end{array}$ \\
\hline \hline${ }^{1} \mathrm{H}$ & positive & 5.8 & 69.98 \\
\cline { 2 - 4 } & negative & -4.0 & 70.22 \\
\hline \hline${ }^{19} \mathrm{~F}$ & positive & 3.3 & 69.95 \\
\cline { 2 - 4 } & negative & -2.7 & 70.30 \\
\hline
\end{tabular}

Table.3: The sumarize of relaxation time $T_{l}$ of ${ }^{1} \mathrm{H}$ and ${ }^{19} \mathrm{~F}$.

\begin{tabular}{|c|c|c|}
\hline Temperature ${ }^{\text {Nuclei }}$ & ${ }^{19} \mathrm{~F}$ & ${ }^{1} \mathrm{H}$ \\
\hline $1.0 \mathrm{~K}$ & $21.8 \mathrm{~min}$ & $1.2 \mathrm{~min}$ \\
\hline $1.6 \mathrm{~K}$ & $6.7 \mathrm{~min}$ & $0.2 \mathrm{~min}$ \\
\hline
\end{tabular}

\section{Conclusion}

In preparation of DNP samples, chemical-doping and radiation-doping have been employed widely. The former has restrictions in the choice of the target material and free radicals. The latter requires an electron linac and careful adjustment of the irradiation. A new method called 'mechanical-doping", which has no restriction in choice of a solid sample, has been proposed. A DNP test was performed using the mechanical-doped $\mathrm{LaF}_{3}$ mixed with TEMPO dissolved in ethanol. ${ }^{19} \mathrm{~F}$ polarizations of $3.3 \%$ and $-2.7 \%$ were achieved. Although they are not satisfactorily high, they were as high as about $2 / 3$ of those for ${ }^{1} \mathrm{H}$ in ethanol. The observation of the enhancement of ${ }^{19} \mathrm{~F}$ polarization proves the effectiveness of the mechanical-doping for DNP. 


\section{References}

[1] D.G.Crabb and W.Meyer, Annu. Rev. Nucl. Part. Sci. 199747:67-109

[2] W.Meyer, NIM A, 526 (2004) 12-21

[3] M.Pl“ uckthun et al., NIM A, 400 (1997) 133-136

[4] S1-project of No.2015S12 in neutron science division of KEK 